\title{
Decoupling of helium in the winds of chemically peculiar stars
}

\author{
Jiří Kubát \\ Astronomický ústav AV ČR, CZ-251 65 Ondřejov, Czech Republic \\ Jiří Krtička \\ ÚTFA PřF MU, Kotlársská 2, CZ-611 37 Brno, Czech Republic
}

\begin{abstract}
We calculate hydrodynamic multicomponent models of radiatively driven winds in B type stars. Our wind models consist of four components, namely hydrogen, helium, accelerated ions, and electrons. We solve equations of continuity, motion, and energy for all components and we take into account mutual collisions. The resulting models show large heating caused by friction. Results of our calculations show that the explanation of helium chemical peculiarity by the helium decoupling from the mean wind is unlikely.
\end{abstract}

\section{Introduction}

It is generally believed that peculiar abundances of chemical elements in stellar atmospheres are caused by diffusion processes (for a review, see Alecian 1995 or Vauclair 2003). However, the influence of a stellar wind should not be overlooked. Decoupling of individual elements in a stellar wind may lead to their leakage from the stellar atmosphere. This may serve as an additional mechanism of creating peculiar abundances in B and A stars. Based on hydrodynamical calculations of Babel (1996), Hunger \& Groote (1999) suggested the idea that the fractionated wind may cause He abundance anomalies in B stars.

Here we present a solution of the hydrodynamical equations which may lead to a fractionated stellar wind. Preliminary test calculations have already been shown in Krtička \& Kubát (2001).

\section{The physical model}

The line radiatively driven stellar wind in our model is described as a four component gas consisting of accelerated ions, hydrogen, helium, and electrons. For each component we solve a set of hydrodynamic equations ( $a$ and $b$ indicate particular components - accelerated ions, hydrogen, helium, and electrons), namely the continuity equation

$$
\frac{1}{r^{2}} \frac{d}{d r}\left(r^{2} \rho_{a} v_{r a}\right)=0
$$


the momentum equation

$$
v_{r a} \frac{d v_{r a}}{d r}=g_{a}^{\mathrm{rad}}-g-\frac{1}{\rho_{a}} \frac{d}{d r}\left(a_{a}^{2} \rho_{a}\right)+\frac{q_{a}}{m_{a}} E+\frac{1}{\rho_{a}} \sum_{b \neq a} k_{a b} G\left(x_{a b}\right) \frac{v_{r b}-v_{r a}}{\left|v_{r b}-v_{r a}\right|},
$$

and the energy equation

$$
\begin{aligned}
& \frac{3}{2} k v_{r a} \frac{\rho_{a}}{m_{a}} \frac{d T_{a}}{d r}+a_{a}^{2} \rho_{a} \frac{1}{r^{2}} \frac{d}{d r}\left(r^{2} v_{r a}\right)=Q_{a}^{\mathrm{rad}}+ \\
& +\frac{1}{\sqrt{\pi}} \sum_{b \neq a} k_{a b} \frac{2 k\left(T_{b}-T_{a}\right)}{m_{a}+m_{b}} \frac{\exp \left(-x_{a b}^{2}\right)}{\alpha_{a b}}+\sum_{b \neq a} \frac{m_{b}}{m_{a}+m_{b}} k_{a b} G\left(x_{a b}\right)\left|v_{r b}-v_{r a}\right| .
\end{aligned}
$$

The equations are solved using the complete linearization method (see Krtička $2001,2003)$.

\subsection{Frictional coupling and decoupling}

The most important term, which determines whether the decoupling is present or not, is the frictional term. Mutual collisions of particles assure transfer of momentum between them. The Coulomb collisions, which are the main interaction mechanism between charged particles, depend heavily on a charge (cf. Eq. 5).

The momentum is transferred from the metallic ions (accelerated by line absorption) to non-accelerated (passive) atoms of hydrogen and helium via Coulomb collisions that give rise to a frictional force,

$$
R_{a b}=-n_{a} n_{b} k_{a b} G\left(x_{a b}\right),
$$

where $n_{a}, n_{b}$ are number densities of individual components,

$$
k_{a b}=\frac{4 \pi(\ln \Lambda) q_{a}^{2} q_{b}^{2}}{k T_{a b}} \frac{v_{r a}-v_{r b}}{\left|v_{r a}-v_{r b}\right|}
$$

$k_{a b}$ is the friction coefficient, $q_{a}, q_{b}$ are charges of individual components, $T_{a b}=$ $\left(m_{a} T_{b}+m_{b} T_{a}\right) /\left(m_{a}+m_{b}\right), x_{a b}=\left|v_{r a}-v_{r b}\right| / \alpha_{a b}$ is the velocity difference in thermal units, $\alpha_{a b}^{2}=2 k\left(m_{a} T_{b}+m_{b} T_{a}\right) /\left(m_{a} m_{b}\right), v_{r a}, v_{r b}$ are velocities of individual components, and $G\left(x_{a b}\right)$ is the Chandrasekhar function.

The amount of momentum transferred by Coulomb collisions depends heavily on the charge of the colliding particles and their densities. Therefore, for $\mathrm{O}$ and hot B stars, where the ionization degree is high, friction is large, and no decoupling in the wind occurs.

On the other hand, for weaker winds of cooler B stars the ionization degree is lower, and, consequently, friction is also lower. This makes the effect of decoupling possible. However, as was found by Krtička \& Kubát (2000), decoupling does not always occur in these winds, sometimes only a significant change of wind dynamics takes place. Conditions for decoupling are more subtle due to the nonlinear dependence of the radiative acceleration on the velocity gradient. Alternatively, the multicomponent flow may become unstable in this case (Krtička \& Kubát 2002, Owocki \& Puls 2002). 

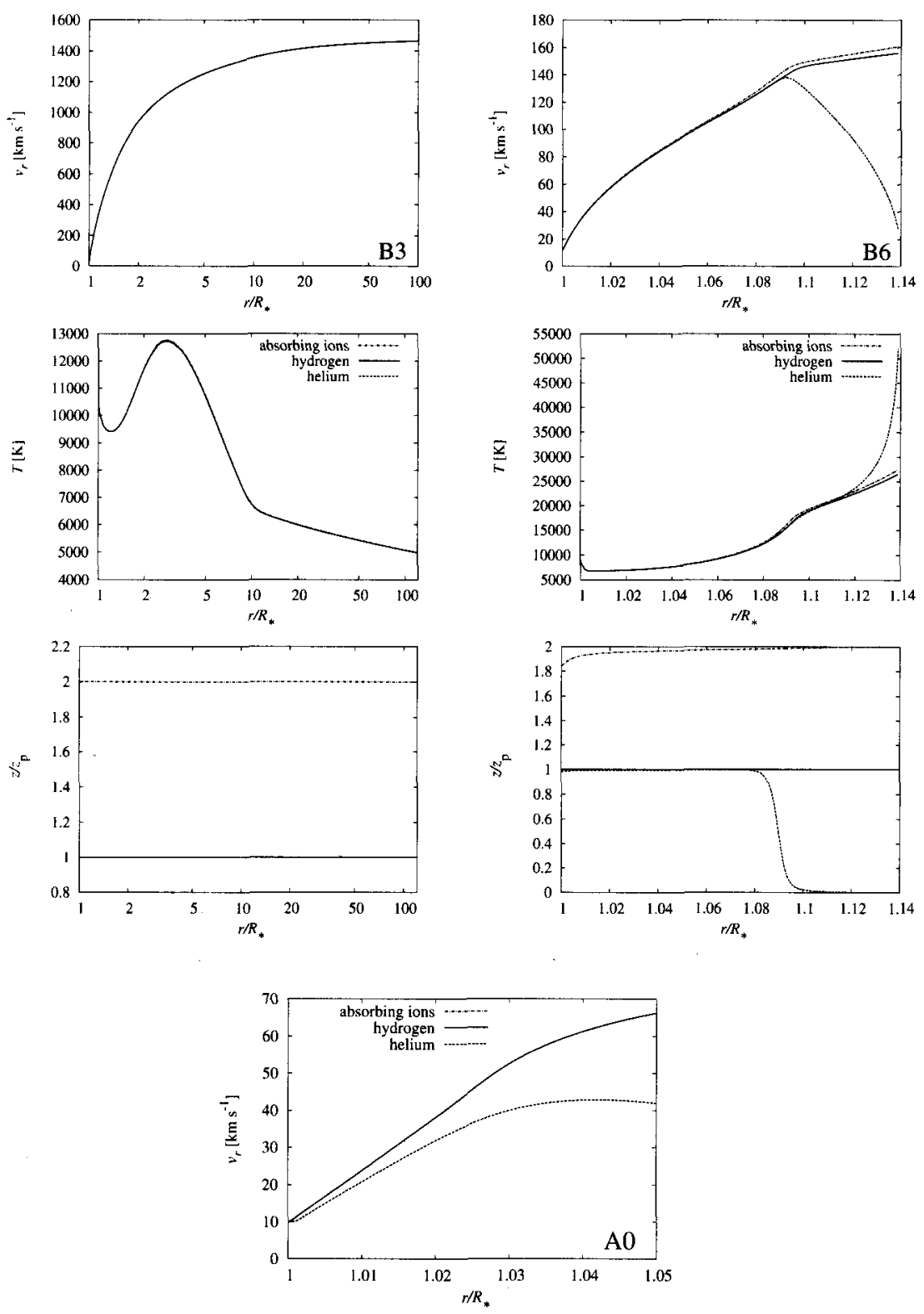

Figure 1. Four-component winds from main sequence B3 and B6 stars. Panels in the first row display the velocity profile of the wind, the second row panels show the temperature structure of the frictionally heated wind, the third row panels display the charge of individual components, and the lowermost panel displays the velocity profile of an A0 supergiant ( $T_{\text {eff }}=10000 \mathrm{~K}, R=70 \mathrm{R}_{\odot}$, and $\left.M=15 \mathrm{M}_{\odot}\right)$. 


\section{Winds from main sequence stars and supergiants}

The case of a wind from main sequence stars is shown in Fig. 1. For the case of a B3 main sequence star ( $\left.T_{\text {eff }}=19100 \mathrm{~K}, \log g=4.12\right)$, friction is effective due to a high charge of all components and causes only little heating and no decoupling of the wind components occurs. However, starting from a B6 main sequence stellar type ( $\left.T_{\text {eff }}=14100 \mathrm{~K}, \log g=4.12\right)$, in addition to heating, helium decouples since helium charge is low, and may fall back to the stellar surface (see also Porter \& Skouza 1999). As a consequence, we obtain a helium-free wind which may lead to an overabundance of helium in a stellar atmosphere. However, since He-rich stars are observed mainly among early B type Stars, we conclude that the explanation of helium overabundance by the helium decoupling suggested by Hunger \& Groote (1999) is unlikely.

In addition to the winds from main sequence stars, we calculated multicomponent wind models from supergiants. These stellar winds have different ionization and density structure compared to the main sequence wind models, thus the helium decoupling occurs for different stellar effective temperatures. As an example, we present a multicomponent wind model for a star with $T_{\text {eff }}=10000 \mathrm{~K}$, $R=70 \mathrm{R}_{\odot}$, and $M=15 \mathrm{M}_{\odot}$ (an A0 supergiant). A clear decoupling of helium was found. By detailed analysis the boundary between helium coupling and decoupling was estimated at $T_{\text {eff }}=10700 \mathrm{~K}$ for A supergiants.

\section{Conclusions}

For cool B stars, decoupling of a radiatively driven wind is possible. It may lead to selective mass-loss and cause abundance anomalies. However, the explanation of helium overabundance by its decoupling is doubtful.

Acknowledgments. This work was supported by grants 205/01/0656 and 205/02/0445 (GA ČR). The Astronomical Institute Ondřejov is supported by projects K2043105 and Z1003909.

\section{References}

Alecian, G. 1995, Astrofizika, 38, 533

Babel, J. 1996, A\&A, 309, 867

Hunger, K., Groote, D. 1999, A\&A, 351, 554

Krtička, J. 2001, Ph.D. thesis, Masaryk University Brno

Krtička, J. 2003, in ASP Conf. Ser., Vol. 288, Stellar Atmosphere Modelling, eds. I. Hubeny, D. Mihalas \& K. Werner; (San Francisco ASP), 259

Krtička, J., Kubát, J. 2000, A\&A, 359, 983

Krtička, J., Kubát, J. 2001, A\&A, 369, 222

Krtička, J., Kubát, J. 2002, A\&A, 388, 531

Owocki, S. P., Puls, J. 2002, ApJ, 568, 965

Porter, J.M., Skouza, B.A. 1999, A\&A, 344, 205

Vauclair, S. 2003, Ap\&SS, 284, 205 